\title{
Sharpe Thinking with Asymmetrical Preferences
}

\author{
Simone Farinelli* and Luisa Tibiletti ${ }^{\dagger}$
}

October 9th, 2002

\begin{abstract}
As we leave behind the assumption of normality in return distributions, the classical risk-reward Sharpe Ratio becomes a questionable tool for ranking risky projects. In the spirit of Sharpe thinking, a more general risk-reward ratio $\Phi$ suitable to compare skewed return distributions with respect to a benchmark, is introduced. This index captures two types of asymmetry information: (1) "good" volatility (above the benchmark) and "bad" volatility (below the benchmark) are differently weighted, (2) asymmetrical preferences to "small" and "large" deviations from the benchmark are modelled. The former goal is achieved by using one-sided volatility measures, and the latter by choosing appropriate order for the one-sided moments involved. The Omega Index (see Cascon et al. (2002) and the Upside Potential Ratio (see Sortino (2000) follow as special cases of the index $\Phi$. Moreover, compatibility of the ranking rule based on ratio $\Phi$ with the expected utility framework is proved.
\end{abstract}

JEL Subject Classification: G0,G1,G2.

Key Words: Sharpe Index, One-Sided Risk Measures, First Order Risk Aversion.

*Quantitative and Bond Research, Cantonal Bank of Zurich, P.O. Box, CH-8010 Zurich, Switzerland; e-mail: simone.farinelli@zkb.ch

†Dipartimento di Statistica e Matematica, Università di Torino, Piazza Arbarello, 8, 10122 Torino, Italy; e-mail:luisa.tibiletti@unito.it 


\section{Introduction ${ }^{1}$}

More than thirty-five years ago, Sharpe [Sha66] introduced a performance measure for mutual funds, originally termed as reward-to-variability. Subsequently, under the name of Sharpe Ratio, it has became one of the most popular index in academic research and in Financial Management practical applications. In recent years, the growing compelling requirement to express performance of risky projects with respect to a benchmark $b$, has lead to a wider interpretation of this ratio (see [Sha94]): the Sharpe Ratio of any investment $X$ is simply defined as the expected excess return $(X-b)$ over a benchmark $b$ divided by the corresponding standard deviation. Although this ratio is fully compatible with normally distributed returns (or, in general, with elliptical returns), it may lose reliability in interpreting risk adjusted performance as we relax this artificial assumption (see [Le99] and $\left.[\mathrm{BeLe} 00]^{2}\right)$. In an asymmetrical world, the "good" volatility (above the benchmark) and "bad" volatility (below the benchmark) of $(X-b)$ may be strongly different. The question to ask is whether we are interested in separate information about them or rather in a global one. The answer depends on the goal. If our aim is pricing a risky asset, where we focus on capturing the "stability" around a "central tendency", then a global "two-sided" information is required. In this case, a two-sided volatility measure, as the standard deviation, hits the goal. But a different perspective may be of our interest. In recent normative requirements, prevention to risk default has become a compelling point. Think of the worldwide explosion of credit derivative products like Credit Default Swaps and Credit Linked Notes since 1996. Another current hot topic is the responsibility of risk managers to guarantee the profitability of mutual and pension funds, as well as to meet the future liabilities keeping the funding ratio above the solvability constraints over a long term time horizon. In all above cited cases, all attention is focused on the "left-sided" variability. So, as all we are concerned about is falling below (or above) a benchmark $b$, one-sided measures are the right tools to use (see [GKD02] ). In conclusion, the classical Sharpe Index based on the most common two sided variability measures, the expected value (for the "favorable" volatility) and the standard deviation (for the "unfavorable" volatility), may be inappropriate in this context.

The paper at hand heads a step beyond this classical approach in order to handle with

\footnotetext{
${ }^{1}$ We would like to thank Lucio Bertoli-Barsotti and Alessandra Durio for their valuable comments and suggestions. We are also grateful to Con Keating for a stimulating discussion on the topic. The views expressed herein are those of the authors and do not necessarily reflect opinions shared by Cantonal Bank of Zurich.

${ }^{2}$ Bernardo and Ledoit ([BeLe00]) to overcome the problem propose a semi-parametric alternative based on the gain-loss ratio that cuts out the extremal events on the tails of the distribution.
} 
asymmetrical return distributions and enhance asymmetrical preferences. The target is hit by a new risk-reward index $\Phi_{b}$ (see Definition 2.1). A double type of asymmetrical preferences can be modelled:

- asymmetry in preference to "good" and "bad" volatility from the benchmark,

- asymmetry in preference to "small" and "large" deviations from the benchmark.

The former asymmetrical attitude is captured by using one-sided volatility measures. But additional advantages in using one-sided risk measures exist. Among the others they are convex measures capturing the spontaneous idea that portfolio diversification tends to shrink the extremal events on both distribution tails. Moreover, upper and lower bounds for the index involving an asset portfolio are easily carried out in terms of the correspondent one-sided moments of the assets.

The latter asymmetrical attitude is described by the orders of the one-sided moments involved. The higher the order is, the higher the agent's inclination (in the case of expected gains) or dislike (in the case of expected losses) for the extremal events. Therefore, if moderate deviations from the benchmark are relatively harmless when compared to large deviations, then a high order for the left-sided moment is suitable. Vice versa, if small successful outcomes over the benchmark are relatively appreciated with respect to exceptional large stakes, then a low order for the right-sided moment well fits the purpose.

As expected, we prove that the ranking rule based on the performance index $\Phi_{b}$ is benchmark dependent. As intuition suggests, for a fixed the risky project $X$, as the benchmark $b$ increases the performance index $\Phi_{b}$ decreases. For a glance at the sensitivity of the performance to a benchmark shift, the elasticity of $\Phi_{b}$ at $b$ is worked out.

A further point deserves a thoroughly insight. We show that a decision-making rule based on $\Phi_{b}$ index is fully consistent with the expected utility theory. The representative agent with asymmetrical preferences towards "good" and "bad" variability displays firstorder risk aversion according to Segal-Spivak's [SeSp90] definition in correspondence to the benchmark $b$. The associated utility function is a piecewise polynomial. Its degree depends on the preference to extremal events on the left and right tails of the distributions.

The paper is organized as follows. In Section 2 the properties of the one-sided variability measures are explored. In Section 3 the performance index $\Phi_{b}$ is introduced. Then absolute and relative sensitivity to the benchmark is evaluated. In Section 4 the selection rule based one-sided moments is proved to be compatible with the expected utility framework. A conclusion in Section 5 ends the note. 


\section{One-sided variability measures}

Let consider an asset with random total return over a certain period, denoted by $X$. Its performance is to be measured in comparison with a reference target $b$. In general, the benchmark is an exogenous data that reflects the context and may vary with the time. It could be the liability return for a pension fund, the total return of benchmark portfolios for traditional asset managers, or just the money market rates for hedge funds. The variable under consideration becomes the excess ${ }^{3}$ return $(X-b)$. We assume that $X$ is integrable, i.e., $\mathrm{E}[|X|]<\infty$. If necessary, we also assume the existence of all higher moments up to the order required.

We introduce the notation $\xi^{-}:=-\min \{\xi, 0\}$ and $\xi^{+}=\max \{0, \xi\}$ and the following

Definition 2.1 Let a benchmark $b \in \mathbf{R}$ and $p>0$ be fixed. The $p$-th partial moments of the excess return $X-b$

$$
m_{-, b}^{p}(X):=E\left[\left\{(X-b)^{-}\right\}^{p}\right] \quad m_{+, b}^{p}(X):=E\left[\left\{(X-b)^{+}\right\}^{p}\right]
$$

are called, respectively, the left-sided moment and the right-sided moment of $p$-th order for the excess return of $X$ with reference to the benchmark $b$.

The left-sided moments are loss risk measures, whereas the right-sided ones are gain variability measures.

\subsubsection{Properties of one-sided moments}

The use of left-sided moments is not new in finance. As a matter of fact the focus on downside risk is not only sensible and theoretically founded but it can even be traced back to Markowitz [Mar52] and Roy [Ro52] (for a historical survey see [Na99]). Fishburn [Fn77] uses them as risk measures in a mean-risk analysis frame proving compatibility with expected utility theory. A set of properties are collected below:

- one-sided $p$-th order moments are always non-negative, for $p>0$.

- Clearly, the one-side moments are not positive homogeneous measures, and thus not scale invariant. This drawback can be easily overcome by the following normalization:

$$
\rho_{-, b}^{p}(X):=E^{1 / p}\left[\left\{(X-b)^{-}\right\}^{p}\right] \quad \rho_{+, b}^{p}(X):=E^{1 / p}\left[\left\{(X-b)^{+}\right\}^{p}\right] .
$$

\footnotetext{
${ }^{3}$ Sharpe [Sha66] considered the return $R$, instead to the excess of return $X=R-b$. Note that we can come back to this original definition by setting $b=0$.
} 
Note that $\rho_{-, b}^{2}(X)$ with $b=E[X]$ coincides with the well-known semi-standard deviation.

- $\rho_{-, b}^{p}$ and $\rho_{+, b}^{p}$ are coherent risk measures (see [Ti02] and [Fr02]) and, in particular, they both enjoy sub-additivity.

- Sub-additivity captures the intuitive effects of diversifications of the portfolio. The rationale behind sub-additivity can be summarized by the statement "a merger does not create extra risk" (see [ADEH99] page 209), and if applied to favorable variability, "does not create extra return". In other words, diversification tends to shrink both the extreme tails of the distribution: no extra losses and no extra gains are created!

Remark 2.2 How to choose the proper order $p$ to use? The choice depends on the desired relevance given to the magnitude of deviations: the higher $p$, the more emphasis is given to the outcomes on the tail involved. In other words, to highlight extremal events, one has just to select a higher order p. Examples will be given in the next section.

As far as a two-sided variability measure is concerned, a way to capture the presence of extremal events in an asset return distribution is to look at its higher moments. For example, the skewness is provided by a properly normalized third moment. Positive skewness means that the right tail of the distribution supports a greater probability mass than the left one. Despite of this clear interpretation, the step from the analysis of single asset to that of a portfolio is a puzzling task. Intuition suggests that some elementary properties of the assets' return should be preserved under linear combination. Unfortunately this conjecture turns out to be false: with the exception of elliptical distributions not even a portfolio of identical assets inherits their characteristics. It can be proved that even null correlated identically distributed assets may switch in skewness direction as they are composed in the portfolio. The real "culprit" of this embarrassing drawback is the overwhelming presence of the mixed moments in the calculations of the higher moments. Since both unfavorable and favorable events for all assets contribute to the portfolio return distribution, not only the magnitude of portfolio moments but even their sign (in the odd order case) may have no relation with the ones of the portfolio assets (see [Ti02] for a deeper discussion on the topic). Moreover, no bounds for the higher moments are available. On the contrary, this kind of problems does not arise when using one sided measures, and clear-cut upper bounds for the measures $\rho_{-, b}^{p}\left(\right.$ and $\left.\rho_{+, b}^{p}\right)$ in terms of the corresponding $\rho_{-, b}^{p}$ (and $\rho_{+, b}^{p}$ ) of the addenda can be easily worked out. 
Proposition 2.3 Given the portfolio $S=\sum_{i=1}^{n} c_{n} X_{n}$ of risky assets $X_{i}$ and weights $c_{i} \in \mathbf{R},, i=1, . . n$, following lower and upper bounds hold

$$
\begin{aligned}
& 0 \leq \rho_{-, b}^{p}(S) \leq \sum_{i=1}^{n}\left|c_{n}\right| \rho_{-, b}^{p}\left(X_{n}\right) \\
& 0 \leq \rho_{+, b}^{p}(S) \leq \sum_{i=1}^{n}\left|c_{n}\right| \rho_{+, b}^{p}\left(X_{n}\right) .
\end{aligned}
$$

PROOF: By Minkowski's inequality and convexity of the operators $(\cdot)^{+}$and $(\cdot)^{-}$.

\section{The one-sided variability ratio $\Phi_{b}$}

Let $I:=\bar{\Im}(X)$ be the support of $X$ and $b$ an interior point of the convex hull of $I$. This simply means that a probability mass exists on the left of the benchmark, as it is sensible to be. Existence and finiteness of the one sided moments involved ${ }^{4}$ is also required.

Definition 3.1 The performance ratio based on one sided risk measures of an asset with total return $X$ and benchmark $b$ is defined for any $p, q>0$ using its excess return $X-b$ as:

$$
\Phi_{b}^{p, q}(X):=\frac{E^{1 / p}\left[\left\{(X-b)^{+}\right\}^{p}\right]}{E^{1 / q}\left[\left\{(X-b)^{-}\right\}^{q}\right]}
$$

The positive real numbers $p$ and $q$ are called the right and respectively left orders of the performance ratio.

Remark 3.2 The $\Phi_{b}$ is nothing but the ratio between the favorable events (properly weighted) and the unfavorable ones. It can be seen just as the reward-to-variability, or, in economic terms, as the shadow price for unit of risk for the excess return

Remark 3.3 As the benchmark $b$ is fixed, the higher the index $\Phi_{b}^{p, q}$, the more preferable is the risky trade $X$. This is a selection rule providing preference ranking for a set of comparable trades, that is, having the same benchmark.

\footnotetext{
${ }^{4}$ The goal is achieved by assuming a finite support $I$ for $X$. As we are modelling real financial data, this is a realistic assumption. Anyway, looser condition can stressed out.
} 
Remark 3.4 As mentioned before, the higher the orders $p$ and $q$ are, the more emphasis is given to the extremal events on the distribution tails. For a given benchmark $b$, the left order $q$ is supposed to reflect agent's feeling about the relative consequences of falling below $b$ by various amounts. If the agent's main concern is failure to hit the target without particular regard to the amount, then a small value (i.e. $q<<1$ ) for the left order is appropriate. On the other hand, if small deviations below the benchmark are relatively harmless when compared to large deviations (catastrophical events), then a large value (i.e. $q>>1$ ) for the left order is advisable. An analogous reasoning can be stressed for the choice of the proper right order $p$. It captures the relative appreciation for outcomes above the benchmark. If moderate gains are relatively highly appreciated with respect to exceptional performance, a low right order (i.e. $p<<1$ ) is indicated. Vice versa, an high $p>1$ is suggested to describe the opposite attitude.

Remark 3.5 In the context of pension fund performance measurement Sortino [So00] defined the upside potential ratio

$$
R(X):=\frac{E\left[(X-M A R)^{+}\right]}{\sqrt{E\left[\left\{(X-M A R)^{-}\right\}^{2}\right]}} .
$$

where MAR is the minimum acceptable return. It is worthwhile noting that $R(X)$ can be seen as a special case of $\Phi_{b}^{p, q}$, as soon as we fix $b=M A R$ and the $p=1, q=2$.

Example 3.6 A special case of symmetrical preference to small and large deviations from the benchmark: the Omega Index.

Let $p=q:=1$. The index $\Phi_{b}^{p, q}$ reduces to

$$
\Omega_{b}(X)=\frac{E\left[(X-b)^{+}\right]}{E\left[(X-b)^{-}\right]} .
$$

an index introduced in [CKS02]. ${ }^{5}$ This index has a very natural interpretation: it is the ratio between the expected gains and the expected losses. Due to a non sharp preference in extremal events (both favorable and unfavorable), $\Omega$ may turn out to be a smooth

\footnotetext{
${ }^{5}$ [CKS02] defined

$\Omega_{b}(X):=\frac{\int_{b}^{+\infty}[1-F(x)] d x}{\int_{-\infty}^{b} F(x) d x}$

where $F$ is the distribution function of $X$. Since $E[\max (0, X-b)]=\int_{b}^{+\infty}[1-F(x)] d x=E\left[(X-b)^{+}\right]$ and $E[\min (0, X-b)]=\int_{-\infty}^{b} F(x) d x=E\left[(X-b)^{-}\right]$

equivalence between the two of the definitions is proved.
} 
water-shed for discriminating differently distributed assets. Let us consider the following asset total returns:

$$
X(\omega)=\left\{\begin{array}{cc}
3 & \text { if } \omega=\frac{1}{3} \\
4 & \text { if } \omega=\frac{1}{3} \\
11 & \text { if } \omega=\frac{1}{3}
\end{array} \quad Y(\omega)= \begin{cases}1 & \text { if } \omega=\frac{1}{3} \\
8 & \text { if } \omega=\frac{1}{3} \\
9 & \text { if } \omega=\frac{1}{3} .\end{cases}\right.
$$

Let $b=6$, so the excess of returns become

$$
(X(\omega)-6)=\left\{\begin{array}{lll}
-3 & \text { if } & \omega=\frac{1}{3} \\
-2 & \text { if } & \omega=\frac{1}{3} \\
+5 & \text { if } & \omega=\frac{1}{3}
\end{array} \quad(Y(\omega)-6)=\left\{\begin{array}{lll}
-5 & \text { if } & \omega=\frac{1}{3} \\
+2 & \text { if } & \omega=\frac{1}{3} \\
+3 & \text { if } & \omega=\frac{1}{3}
\end{array}\right.\right.
$$

clearly, they display same mean and variance. Straightforward calculations prove $\Omega_{6}(X)=$ $\Omega_{6}(Y)=1$, so $X$ and $Y$ turn out to be indifferent under $\Omega_{6}$.

Nevertheless, the trades under comparison show different risk-reward features. As $X$ displays "moderate" unfavorable outcomes $(x=-3$, and $x=-2)$ and on the other hand the chance of one high stake $(x=+5)$ whereas the trade $Y$ displays "moderate" favorable outcomes $(x=+2$ and $x=+3)$ and a single relevant loss $(x=-5)$. In conclusion, since $p=q=1$, a symmetrical preference has been expressed over the tails. So, in line with the preferences declared, with $b=6, \Omega$ is a dull discriminating tool.

Remark 3.7 The orders $p$ and $q$ do not need to be necessarily equal. On the contrary, an asymmetrical preference on the extremal favorable and unfavorable events is quite normal in real life decisions. An example may cut short any perplexities.

Example 3.8 Asymmetrical preference to small and large deviations from the benchmark

- Suppose one dislikes the chance of having huge losses (even though with low probability), then $p<q$ is fixed. For instance let $p=1$ and $q=2$. For the Example 3.6, we get

$$
\Phi_{b}^{p, q}(X)=\frac{E\left[(X-b)^{+}\right]}{\sqrt{E\left[\left\{(X-b)^{-}\right\}^{2}\right]}}=\frac{\frac{5}{3}}{\sqrt{\frac{13}{3}}} \quad \text { and } \quad \Phi_{b}^{p, q}(Y)=\frac{E\left[(Y-b)^{+}\right]}{\sqrt{E\left[\left\{(Y-b)^{-}\right\}^{2}\right]}}=\frac{\frac{5}{3}}{\sqrt{\frac{25}{3}}}
$$


Therefore, $\Phi_{b}^{p, q}(X)>\Phi_{b}^{p, q}(Y)$ so $X$ should be preferred to $Y$. A coherent policy with the declared preferences is suggested.

- Suppose, now, that the financial policy gives different advises: no care of losses (both small and large deviations from the benchmark), but good chances to highly overcome the benchmark are desirable. Let $p>q$ be fixed. For example, $p=2$ and $q=1:$

$$
\Phi_{b}^{p, q}(X)=\frac{\sqrt{E\left[\left\{(X-b)^{+}\right\}^{2}\right]}}{E\left[(X-b)^{-}\right]}=\frac{\sqrt{\frac{25}{3}}}{\frac{5}{3}} \quad \text { and } \quad \Phi_{b}^{p, q}(Y)=\frac{\sqrt{E\left[(Y-b)^{+}\right]^{2}}}{E\left[(Y-b)^{-}\right]}=\frac{\sqrt{\frac{13}{3}}}{\frac{5}{3}}
$$

Again, since $\left.\Phi_{b}^{p, q}(X)\right)>\Phi_{b}^{p, q}(Y), X$ should be preferred to $Y$. Again, coherence with the financial advises turns out.

- Suppose, different preferences are declared: no care of large deviations from below the benchmark, but very good chances of overcoming (no matter if that happens with small successful) the benchmark are required. Let $p<q$ be fixed. For example, $p=\frac{1}{2}$ and $q=1$ :

$$
\Phi_{b}^{p, q}(X)=\frac{\left(E\left[\sqrt{(X-b)^{+}}\right]\right)^{2}}{E\left[(X-b)^{-}\right]}=\frac{1}{3} \quad \text { and } \quad \Phi_{b}^{p, q}(Y) \frac{\left(E\left[\sqrt{(Y-b)^{+}}\right]^{2}\right)}{E\left[(Y-b)^{-}\right]}=\frac{(\sqrt{2}+\sqrt{3})^{2}}{15}
$$

Again, $\Phi_{b}^{p, q}(X)>\Phi_{b}^{p, q}(Y)$ then $X$ should be preferred to $Y$. Again, coherence with the financial advises turns out.

Remark 3.9 It is worthwhile noting how the classical Sharpe Ratio should be a dull tool for discriminating differently distributed trades. Excess returns $(X-b)$ and $(Y-b)$ in Example 3.6 have the same (null) mean and the same standard deviation. Sharpe index is equal to zero: no discrimination between so different assets is signaled! Sharpe index qualifies $X$ and $Y$ indifferent with respect to the benchmark $b=6$. This result is not surprising at all. The choice of two sided variability measures for both the potential gains and losses expresses a symmetrical preference to negative and positive deviations from the benchmark. Variables under consideration show an "equal" stability from the benchmark. 


\section{Sensitivity to the benchmark}

The choice of the benchmark is an exogenous question with respect to analysis of the performance. The Index $\Phi_{b}^{p, q}$ is of course a function of the benchmark $b$. We want to investigate this functional dependence, in particular by analyzing separately the cases of absolute and relative variations. The latter will lead to the concept of elasticity.

\subsubsection{Absolute Variations of $\Phi_{b}^{p, q}$}

Intuitively the higher the benchmark $b$ is, the lower the possibility of being successful an therefore the higher the benchmark is, the lower the performance index should be. This statement is more precisely way formalized by the following

Proposition 4.1 For given left and right orders $p$ and $q$, the index $\Phi_{b}^{p, q}(X)$ is a decreasing function of the benchmark $b$.

PROOF: Since $E\left\{(X-b)^{+}\right\}^{p}=\int_{b}^{+\infty}(x-b)^{p} d F(x)$ is a decreasing function of $b$, whereas $E\left\{(X-b)^{-}\right\}^{p}=-\int_{-\infty}^{b}(x-b)^{p} d F(x)$ is an increasing function of $b$, the ratio $\Phi_{b}^{p, q}(X)$ turns out to be a decreasing function of the benchmark $b$.

\subsubsection{Relative Variations of $\Phi_{b}^{p, q}$}

For a fixed benchmark $b$, the performance index $\Phi_{b}^{p, q}$ naturally induces a total ordering rule on the set of candidate investments with increasing values of the index. However, Proposition 4.1 proves that the performance index $\Phi_{b}^{p, q}$ decreases for all trades under consideration, as $b$ increases. A preference ordering may be completely modified by a benchmark shift. In a preference ordering framework, the key point is no longer what the absolute impact on $\Phi_{b}^{p, q}$ due to a variation of $b$ is, but what the sensitivity of $\Phi_{b}^{p, q}$ to a benchmark variation. Let us start with an illustrating example.

Example 4.2 Preference ordering induced by the index is not invariant under change of benchmark: Indeed it may be highly sensitive to its variations.

Let us recall Example 3.6 and lower the benchmark to $b=4$. The excess returns become then 


$$
(X(\omega)-4)=\left\{\begin{array}{ccc}
-1 & \text { if } \quad \omega=\frac{1}{3} \\
0 & \text { if } \quad \omega=\frac{1}{3} \\
+7 & \text { if } \quad \omega=\frac{1}{3}
\end{array} \quad(Y(\omega)-4)=\left\{\begin{array}{ccc}
-3 & \text { if } \quad \omega=\frac{1}{3} \\
+4 & \text { if } \quad \omega=\frac{1}{3} \\
+5 & \text { if } \omega=\frac{1}{3}
\end{array}\right.\right.
$$

again, they have the same mean $(=2)$ and the same variance $\left(=\frac{50}{3}\right)$. At this benchmark level, the index $\Omega$ is no longer a dull discriminating index. In fact, $\Omega_{4}(X)=7$ whereas $\Omega_{4}(Y)=3$ and therefore $X$ should be preferred to $Y$.

Remark 4.3 Note that the classical Sharpe index is still equal for both trades, which is - as mentioned before - in line with the choice of two sided variability measures. The two sided "dispersion" of the data is quite similar for both $X$ and $Y$. Vice versa, the use of one sided variability measures, implies asymmetrical preferences to favorable and unfavorable events. In conclusion, with the exception of some special benchmark levels, $\Omega$ is more skewness-sensitive than the Sharpe ratio.

A way to have a glance of the sensitivity of $\Phi_{b}^{p, q}$ with respect to a benchmark shift is to look at its elasticity.

Proposition 4.4 For given left and right orders $q$ and $p$, the elasticity of the index $\Phi_{b}^{p, q}(X)$ at the benchmark $b$ is

$$
\epsilon\left(\Phi_{(\cdot)}^{p, q}(X) ; b\right)=-b\left\{\frac{\int_{b}^{+\infty}(x-b)^{p-1} d F(x)}{\int_{b}^{+\infty}(x-b)^{p} d F(x)}+\frac{\int_{-\infty}^{b}(-(x-b))^{q-1} d F(x)}{\int_{-\infty}^{b}(-(x-b))^{q} d F(x)}\right\}
$$

In the Omega special case, that is with $p=q=1$ the expression for the elasticity becomes

$$
\epsilon\left(\Omega_{(\cdot)}(X) ; b\right)=-b\left\{\frac{1-F(b)}{E(X-b)^{+}}+\frac{F(b)}{E(X-b)^{-}}\right\}
$$

PROOF. Note that

$$
\Phi_{b}^{p, q}(X)=\frac{\sqrt[p]{E\left[\left\{(X-b)^{+}\right\}^{p}\right]}}{\sqrt[q]{E\left[\left\{(X-b)^{-}\right\}^{q}\right]}}=\frac{\sqrt[p]{\int_{b}^{+\infty}(x-b)^{p} d F(x)}}{\sqrt[q]{\int_{-\infty}^{b}(-(x-b))^{q} d F(x)}}
$$

and thus for the first partial derivative of the index with respect to the benchmark

$$
\frac{\partial}{\partial b} \Phi_{b}^{p, q}(X)=-\left\{\frac{\int_{b}^{+\infty}(x-b)^{p-1} d F(x)}{\int_{b}^{+\infty}(x-b)^{p} d F(x)}+\frac{\int_{-\infty}^{b}(-(x-b))^{q-1} d F(x)}{\int_{-\infty}^{b}(-(x-b))^{q} d F(x)}\right\} \Phi_{b}^{p, q}(X)<0
$$


Thus

$$
\begin{aligned}
\epsilon\left(\Phi_{(\cdot)}^{p, q}(X) ; b\right) & =-b\left[\frac{\partial}{\partial b} \log \Phi_{b}^{p, q}(X)\right]= \\
& =-b\left\{\frac{\int_{b}^{+\infty}(x-b)^{p-1} d F(x)}{\int_{b}^{+\infty}(x-b)^{p} d F(x)}+\frac{\int_{-\infty}^{b}(-(x-b))^{q-1} d F(x)}{\int_{-\infty}^{b}(-(x-b))^{q} d F(x)}\right\} .
\end{aligned}
$$

If $p=q=1$, we get the elasticity for $\Omega$ :

$$
\epsilon\left(\Omega_{(\cdot)}(X) ; b\right)=-b\left\{\frac{1-F(b)}{E(X-b)^{+}}+\frac{F(b)}{E(X-b)^{-}}\right\}
$$

Remark 4.5 Note that the elasticity of $\Omega$ is proportional to $\frac{1}{H}=\frac{1-F(b)}{E\left[(X-b)^{+}\right]}+\frac{F(b)}{E\left[(X-b)^{-}\right]}$, where $H$ is the weighted harmonic mean of the expected gains, i.e. $E\left[(X-b)^{+}\right]$and the expected losses, i.e. $E\left[(X-b)^{-}\right]$, with weights $1-F(b)$, i.e., the probability to get above the benchmark, and $F(b)$, i.e., the probability to get below the benchmark. Moreover, if $b$ coincides with the median of $X$, the $\epsilon\left(\Omega_{(\cdot)}(X) ; b\right)=0$ and $\Omega$ is anelastic.

Although at first sight the elasticity may seem to have a cumbersome expression, it may turn out to be a friendly-to-use formula as soon as the return empirical distribution is known.

\section{Expected Utility and One-Sided Measures}

In his cornerstone paper Fishburn [Fn77] extended the seminal mean-target semivariance model mentioned by Markowitz [Mar52] and discussed in detail by Mao[Mao70] and Porter [Po74]. If risk is measured by the left sided moment of $q$-th order, mean-risk ratio is congruent with the expected utility theory of von Neumann and Morgenstern.

Along the same line of thought it is possible to identify analytically a utility function depending only on mean and the left and the right sided moment up to order $q$ and $p$.

Theorem 5.1 Let $b \in \mathbf{R}$ be a fixed benchmark and $\mathcal{A}_{n}$ the set of all random variables $X$ having finite partial moments $m_{-, b}^{j}(X)$ and $m_{+, b}^{j}(X)$ for all positive integers $j \leq n$. If the objective function of an expected utility maximizer on $\mathcal{A}_{n}$ acts only on the basis of mean and on the left and the right sided moment up to order $n$, then the associate utility function has the following form

$$
u_{b}(x)=\left\{\begin{array}{cc}
-\sum_{j=1}^{q} k_{j}|x-b|^{j} & x \leq b \\
\sum_{j=1}^{p} h_{j}(x-b)^{j} & x>b
\end{array}\right.
$$


where $k_{j}$ and $h_{j}$ are non-negative parameters. The expected utility has then the following form for any $X \in \mathcal{A}_{n}$

$$
E\left[u_{b}(X)\right]=-\sum_{j=1}^{q} k_{j} m_{-, b}^{j}(X)+\sum_{j=1}^{p} h_{j} m_{+, b}^{j}(X) .
$$

\section{PROOF:}

By Taylor's expansion formulae from the left and from the right for the utility function $u$ at $b$.

Remark 5.2 Compatibility of $\Phi_{b}^{p, q}$ rule with expected utility.

Let $p$ and $q$ be the right and left moment order. Let

$$
k_{j}=\left\{\begin{array}{ll}
=0 & j \neq q \\
\neq 0 & j=q
\end{array} \quad h_{j}= \begin{cases}=0 & j \neq p \\
\neq 0 & j=p\end{cases}\right.
$$

The expected utility has then the following form

$$
E\left[u_{b}(X)\right]=-k_{q} m_{-, b}^{q}(X)+h_{p} m_{+, b}^{p}(X)=h_{p}\left[m_{+, b}^{p}(X)-\frac{k_{p}}{h_{p}} m_{-, b}^{q}(X)\right]
$$

Since the utility function is defined up to a linear transformation, we conclude that the expected utility can be expressed by a linear combination of two indexes: 1) $m_{+, b}^{p}(X)$ an index of the expected favorable outcomes, 2$) m_{-, b}^{q}(X)$ an index of the expected unfavorable outcomes. Both the indexes are properly "shaped" (by the orders $p$ and $q$, respectively) in order to describe the agent's preference toward the extremal events.

In conclusion, a selection rule based on the $\Phi_{b}^{p, q}$ is fully compatible with the expected utility theory as soon as the preferences are described as above.

Remark 5.3 If for the sensitivities $k \neq h$, the utility function $u_{b}$ displays a kink at $b$. According to Segal-Spivak's definition [SeSp90], the representative agent is said to have first-order risk aversion. Non differentiability means that the sensitivity to get above or below the target is not symmetrical. Empirical tests have proved that the assumption of first-order risk aversion can substantially improve the description of the agent's acting in real life (see [EZ01]).

\section{Conclusion}

In the spirit of Sharpe thinking ([Do01]) a new risk-reward ratio $\Phi$ able to measure the performance relative to a benchmark of skewed risky project, is introduced. Two 
different types of asymmetrical preferences can be modelled: 1) asymmetrical preference to "favorable" and "unfavorable" variability from the benchmark, and 2) asymmetrical preference to "small" and "large" deviations. The former kind of asymmetry is captured by using one-sided variability measures, the latter through an appropriate "weighting" of the order of the one-sided moments involved. The ranking rule based on $\Phi$ is proved to be compatible with the expected utility approach.

\section{References}

[ADEH99] Artzner P., Delbaen F., Eber J. and D. Heath, Coherent Measures of Risk, Mathematical Finance, 9, 3 (1999), 203-228.

[BeLe00] Bernardo A.E. and Ledoit O., Gain, Loss, and Asset Pricing, Journal of Political Economy, 108(1), (2000), 144-172.

[CKS02] Cascon A., Keating C. and W. Shadwick, An Introduction to Omega, The Finance Development Centre, 2002.

[Do00] Dowd, K. , Adjusting for Risk: An Improved Sharpe Ratio, International Review of Economics and Finance, 9 (2000),209-222.

[Do01] Dowd, K., Sharpe thinking, Risk, Risk Management for Investors, Special Report, June (2001), S22-S24.

[EZ90] Epstein L.G. and S.E. Zin, First-Order Risk Aversion and the Equity Premium Puzzle, Journal of Monetary Economics, 26(3),(1990), 387-407.

[EZ01] Epstein L.G. and S.E. Zin, The independence axiom and the asset returns, Journal of Empirical Finance, 8, (2001), 537-572.

[Fr02] Fischer, T., Risk Capital Allocation by Coherent Risk Measures based on OneSided Moments, presented at the Sixth International Congress on Insurance Mathematics and Economics, Lisbon, July 15-17, 2002. Text downloadable from http://pascal.iseg.utl.pt/ cemapre/ime2002/

[Fn77] Fishburn, P.C., Mean-Risk Analysis with Risk Associated with Below-Target Returns, American Economic Review, 67, (1977), 116-126.

[GKD02] Goovaerts M.J., Kaas R. and J. Dhaene, Economical Capital Allocation Derived from Risk Measures presented at the Sixth International Congress on 
Insurance Mathematics and Economics, Lisbon, July 15-17, 2002. Text downloadable from http://pascal.iseg.utl.pt/ ${ }^{\sim}$ cemapre/ime2002/

[Le99] Leland, H.E., Beyond mean-variance: performance measurement in a nonsymmetrical world, Financial Analyst Journal, vol. 55, 1999, 27-35.

[Na99] Nawrocki, D.N., A Brief History of Downside Risk Measures, Journal of Investing, Fall 1999, 9-26.

[Mao70] Mao, J.C.T. Models of Capital Budgeting, E-V vs. E-S, Journal of Financial and Quantitative Analysis, 4, (1970), 657-675.

[Mar52] Markowitz, H.M., Portfolio Selection, Journal of Finance, 1952, 7(1), 77-91.

[Po74] Porter, R.B., Semivariance and Stochastic Dominance: A Comparison, American Economic Review, 64, (1974), 200-2004.

[RT01] Rabin, M. and R.H. Thaler, Risk Aversion, Journal of Economic perspectives, 15, (2001), 219-232.

[Ro52] Roy, A. D., Safety First and the holding of assets, Econometrica, 1952, 20(3), 431-449.

[SaSo01] Satchel, S.E. and F. Sortino, Managing Downside Risk, ButterworthHeinemann, Oxford (2001).

[Sha66] Sharpe, W.F., Mutual Funds performance, Journal of Business, January (1966), 119-138.

[Sha94] Sharpe, W.F., The Sharpe Ratio Journal of Portfolio Management, Fall (1994), 49-58.

[PS01] Pedersen C. and S. Satchell, Asymmetric Equilibrium Risk Measures, Journal of Empirical Finance, (2001), forthcoming.

[SeSp90] Segal U. and A. Spivak, First Order Versus second Order Risk Aversion, Journal of Economic Theory, 51(1), (1990), 111-125.

[So00] Sortino, F., Upside-potential ratios vary by investment style, Pensions and Investments, 28(22), (2000), 30-35.

[Ti02] Tibiletti, L., Higher Order Moments and Beyond', presented at the Workshop on Multi-moment Capital Asset Pricing and Related Topics ESCP-EAP European School of Management, Paris, April 29,2002. 